\title{
THE EFFECT OF THERMAL STIMULATION ON THE FAR-RED AND ORANGE-RED IRSL SIGNAL OF A FRENCH K-RICH FELDSPAR: PRELIMINARY RESULTS
}

\author{
MORTEZA FATTAHI ${ }^{1,2}$ \\ ${ }^{I}$ The Institute of Geophysics, The University of Tehran, Kargar Shomali, Tehran, Iran. \\ ${ }^{2}$ The School of Geography, OUCE, South Parks Road Oxford OXI 3QY, U.K.
}

Received 16 January 2009 Accepted 2 September 2009

\begin{abstract}
Feldspar suffers from anomalous fading. Previous studies suggest that the level of fading depends on the emission band, and that the fading decreases by increasing the emission wavelengths; therefore, it is interesting to compare blue, orange and far-red IRSL emission characteristics. The purpose of this article is to make an initial consideration of some physical differences between two close emission windows. The effect of the sample temperature on far-red $(\lambda>650 \mathrm{~nm})$ and orangenear-red IRSL $(\lambda=600-650 \mathrm{~nm})$ from an identical feldspar sample is investigated. The difference in signal decay rate and thermal effects is discussed. While there is signal enhancement for far-red IRSL signals between $100-220^{\circ} \mathrm{C}$, orange-near-red signals do not change. Some possible mechanisms for producing far-red and orange-near-red IRSL are discussed. This paper shows that orange-near-red IRSL and far-red IRSL thermal activation energies are different. This difference may be the cause of previously observed differences in stability of near and far-red IRSL signals.
\end{abstract}

Keywords: IRSL, feldspar, far-red IRSL, orange-near-red IRSL, stimulation temperature.

\section{INTRODUCTION}

Luminescence signal from feldspars offers some advantages over quartz including a higher saturation dose which can enable feldspar to date back to $\sim 1 \mathrm{Ma}$. After irradiation most feldspars display a luminescence $\sim 10-50$ times greater than quartz (e.g., Visocekas et al., 1996). Some feldspars contain a significant proportion of potassium whose radioactive isotope $\left({ }^{40} \mathrm{~K}\right)$ may contribute a significant proportion of the total ionizing radiation dose to the sample. This "internal" dose is unaffected by interstitial water and the thickness of any overburden which could decrease uncertainty in the calculation of the dose rate (Duller, 1997). Satisfactory agreement with independent age control has been shown in some studies (e.g. young samples in Duller, 1994; Clarke et al., 1996); red, blue, and UV emissions can be obtained from an infrared stimulated luminescence (IRSL) signal from feldspars

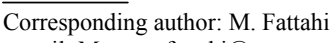

e-mail: Morteza.fattahi@ouce.ox.ac.uk

ISSN 1897-1695 (online), 1733-8387 (print) C 2009 GADAM Centre, Institute of Physics, Silesian University of Technology.

All rights reserved.
(Aitken, 1998). In many circumstances there is either no quartz or the quartz luminescence signal is not suitable for dating, leaving feldspar as the only alternative (e.g., Fattahi et al., 2006). As a result, K-rich feldspar has been widely used as a luminescence dosimeter.

Despite the above mentioned potential advantages of feldspar, the application of infrared stimulation luminescence (IRSL) for dating has been hampered by the widespread observation of anomalous fading and the associated problem of age underestimation (e.g., Lamothe and Auclair, 1999; Richardson et al., 1999; Lian and Shane, 2000; Wallinga et al., 2000).

Previous studies have shown that red thermoluminescence (RTL) and red IRSL (emission $>600 \mathrm{~nm}$ ) show no, or less fading in comparison to the blue luminescence of feldspar (Zink and Visocekas, 1997; Fattahi and Stokes, 2003b). Therefore, the use of far-red and orange-red emissions in IRSL dating of feldspars has the potential to overcome the problem of anomalous fading and may also provide the possibility of extending the dating range up to several $100 \mathrm{ka}$. These advantages of red luminescence attracted the interest of luminescence scientists, and Risø 
included the red luminescence system in its production. Therefore, it is necessary to understand the detailed mechanism of generations of orange-red and far-red IRSL-signals.

It is known that impurities and defects in feldspar result in the appearance of isolated electron levels within the band gap. These can trap electrons or holes. The two hole trapping centers that have been widely investigated are $\mathrm{Mn}^{2+}$ and $\mathrm{Fe}^{3+}$ (Manning, 1970; Telfer and Walker, 1978), which are the main sources of yellow and red luminescence from feldspar.

There are many ways in which defects can participate in luminescence emission. One of these is optically stimulated luminescence (OSL). Hütt et al., (1988) showed that electron transfer from a single defect type dominates IRSL with resonant transition in IR at around $1.4 \mathrm{eV}$. They proposed a model in which the IR stimulates the electron from the ground into an excited state, from where it may be thermally energized into the delocalized band (see McKeever et al., 1997, Fig. 3b). Radioluminescence monitors luminescence emissions arising from relaxation between the excited and grounded state during irradiation, and the dominance of this defect has been confirmed (e.g., Trautmann et al., 2000; Krbetschek et al., 1997). The infrared transitions have, however, been confirmed to be thermally activated in accordance with the Hütt-Jaek-Tchonka (HJT) model, and the thermal activation energy changes from sample to sample from 0.03 to around $0.1 \mathrm{eV}$ (Hütt and Jaek, 1993; Poolton et al., 1995).

Other workers have also reported that the luminescence is thermally enhanced with thermal activation energies in the range 0.02-0.15 eV (e.g. Bailiff and Barnett, 1994; Rieser et al., 1997).

But the thermal activation energy of around $0.15 \mathrm{eV}$ appears still far too small to bridge the energy gap between the excited state and conduction band estimated by Hütt et al. (1988) to be $0.8 \mathrm{eV}$. A detailed study by Poolton et al. (1995) showed that the value of the measured thermal activation energy for OSL in feldspar is dependent upon the optical excitation energy chosen and, in some instances, is much lower than expected. They suggested some possibilities, including:

- directs d-a pair combination from the donor-excited state that requires no thermal activation (may be due to tunnelling from excited state).

- IR stimulates electrons to the conduction band through deep lying band tail states, which requires large thermal activation energy.

Fattahi (2004) combined the measurements of orangenear-red emission during thermoluminescence (RTL), phosphorescence and IRSL to demonstrate the charge recombination mechanisms responsible for the observed orange-near-red IRSL signals from feldspar. He suggested that his observations for orange-near-red IRSL can be interpreted by different mechanisms including tunnelling luminescence processes. It can be argued that tunnelling, in principle, requires no thermal assistance. But this is the case in the occurrence of tunnelling from the excited state. For tunnelling from the ground state of the electron trap there is a minor thermal positioning within the ground state of the electron trap. Visocekas (2000), for example, established that ground-state to ground-state tunnelling could be thermally activated in some cases. Poolton et al. (2002a) argued that although the pure quantum mechanical tunnelling process of the ground state charge cannot be accelerated, the electron wave function can be greatly expanded by the stimulation of electrons into the excited state of the defect. Poolton et al. (2002a) suggested that under low-energy $(1.4 \mathrm{eV})$ optical stimulation, direct electron-hole tunnelling and non-tunnelling (after the excited state electron access the conduction band) could co-exist. They showed that a single component with a low thermal activation energy, around $0.02 \mathrm{eV}$ can be assumed to be the excited-state tunnelling components which suffer from anomalous fading, and a single component with a high thermal activation energy value, can be assumed as a non-tunnelling IRSL components. They believe that if IRSL shows a tunnelling component, then the sample may be subject to fading $(\sim 65 \%$ over a 7-day measurement period); and if the process of producing IRSL is not tunnelling, then the sample will not be subject to fading. They have also shown that the samples with an intermediate thermal activation energy may show some fading $(\sim 10-15 \%$ over a 7 -day measurement period). Molodkov et al. (2007) proposed the direct detection of the tunnel transitions from the dosimetric traps. They suggested that the red tunnel afterglow contains at least two spectrally close components which differ substantially in thermal, kinetic and other properties, and that heating at lower temperatures $\left(100-300^{\circ} \mathrm{C}\right)$ causes changes in many luminescent properties.

Poolton et al. (2002b) suggested that the presence of band-tail states below the conduction band could explain the thermal dependence of IRSL. They argued that the activation energy represented a hopping motion through these tail states. They also found that IRSL decay form of a typical sedimentary K-feldspar is unaffected by sample temperatures while the emission is thermally enhanced (Poolton et al., 2002a, Fig. 6).

Therefore, sample temperature is an important issue in IRSL studies which requires further investigation. This work is an initial attempt to respond to such requirements.

The advance of the current work is to extend the measurements from the orange to far-red band. This paper compares in some detail the temperature dependence of different parts of the orange-near-red and far-red IRSL decay curve from a sedimentary potassium feldspar (laboratory reference $15 / 1)$. The relationship between the initial $(0-2 \mathrm{~s})$, total $(0-100 \mathrm{~s})$, net initial $(0-2 \mathrm{~s}$ less background over $2 \mathrm{~s})$ and net integral $(0-100 \mathrm{~s}$ less background over $100 \mathrm{~s}$ ) laboratory-irradiated IRSL signals versus stimulation temperature $\left(20-460^{\circ} \mathrm{C}\right)$ for preheated $\left(250^{\circ} \mathrm{C}\right.$ for $\left.60 \mathrm{~s}\right)$ samples are investigated. Different processes that affect IRSL signals as a function of sample temperature are discussed.

Special attention is focused on the temperature dependence, separating the effects of different temperaturedependent phenomena, including thermal enhancement and thermal depletion. 


\section{EXPERIMENTAL CONDITIONS}

All measurements were made using a Risø TL-DA-15 automated TL/OSL system (equipped with a ${ }^{90} \mathrm{Sr} /{ }^{90} \mathrm{Y}$ beta source delivering $\sim 7 \mathrm{~Gy} \mathrm{~min}^{-1}$ ) fitted with an IR laser diode emitting at $\lambda=830 \mathrm{~nm}$ as a stimulation source. The intensity of light incident on the sample was about $400 \mathrm{~mW} \mathrm{~cm}{ }^{-2}$ (Bøtter-Jensen et al., 2000). IRSL was detected using a modified Electron Tube D716A "green" bialkaline PMT housed within an S 600 PHOTOCOOL thermoelectric refrigeration chamber, which allows active cooling of the photocathode (operating temperature $\sim 20^{\circ} \mathrm{C}$ ). Luminescence signal emissions were filtered with either an Omega 625 filter (transmission window 600-650 nm), or Schott RG665 + FR400S (transmission window $665-750 \mathrm{~nm}$ ). A full description of the experimental conditions including the detection windows is given in Fattahi and Stokes (2003a). The measurements were made on potassium feldspar $15 / 1$, extracted from fluvial terrace deposits collected from the Loire River in
France (Colls, 1999; Colls et al., 2001). The feldspar grains were separated using standard procedures. The 90$150 \mu \mathrm{m}$ size fractions were mounted as monolayers (approximately $5 \mathrm{mg} /$ per disc) with $10 \mathrm{~mm}$ diameter stainless steel discs using viscosil oil for the single aliquot analyses.

\section{EXPERIMENTAL DESIGN}

The sample was preheated $\left(250^{\circ} \mathrm{C}, 60 \mathrm{~s}\right)$, following laboratory irradiation (240 Gy), and its IRSL decay curves were measured during $100 \mathrm{~s}$ illuminations. The sample was held at temperatures from 40 to $460^{\circ} \mathrm{C}$ in $20^{\circ} \mathrm{C}$ steps during these measurements (Fig. 1 shows selected curves). The aliquot was held at the stimulation temperature for 30 seconds prior to the IRSL measurements to observe possible phosphorescence components and reduce possible thermally stimulated contributions to the IRSL signal. Luminescence was measured at the stimulation temperature, but with no illumination, after
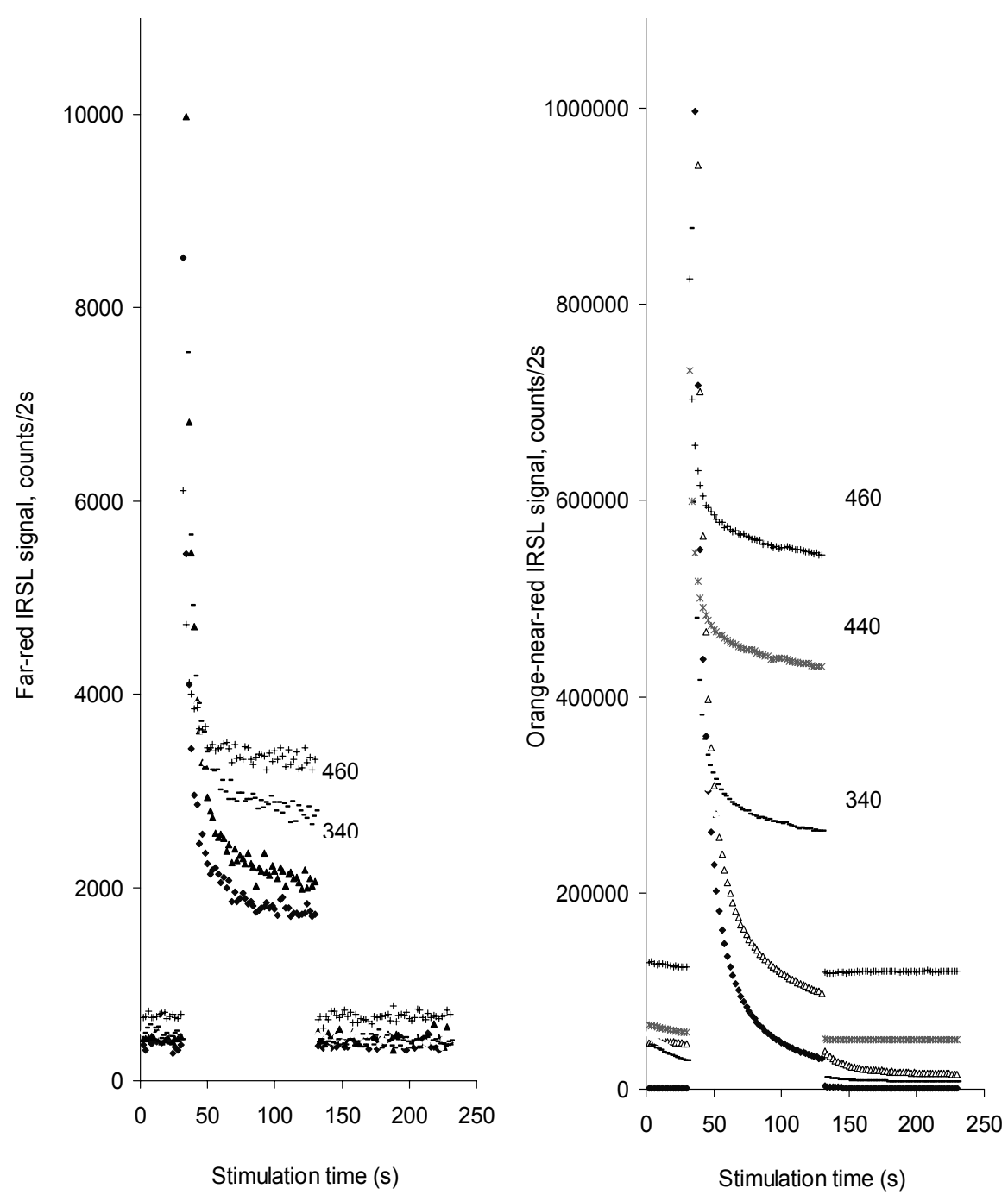

Fig. 1. (a) The far-red IRSL and (b) the orange-near-red IRSL obtained for potassium feldspar (15/1), which had been given a 240 Gy dose, and a 60 $s$ preheat at $250^{\circ} \mathrm{C}$ before every IRSL measurement at elevated temperature from 40 to $460^{\circ} \mathrm{C}$ in $20^{\circ} \mathrm{C}$ steps. To observe possible phosphorescence components and any thermally stimulated contribution to the IRSL signal, the aliquot was held at the stimulation temperature for 30 seconds prior to, and 100 seconds after, the IRSL measurement. 
the IRSL measurement, for a further 100 seconds. This establishes the presence of any thermally stimulated component after $100 \mathrm{~s}$ of illumination. As a result the sample emission was measured for a total time of $230 \mathrm{~s}$ at each temperature. After the luminescence measurement at each sample temperature (from 40 to $460^{\circ} \mathrm{C}$ in $20^{\circ} \mathrm{C}$ steps), a test dose (60 Gy) was given. The sample was then preheated $\left(250^{\circ} \mathrm{C}, 60 \mathrm{~s}\right)$ and its IRSL decay curves were measured during $300 \mathrm{~s}$ illuminations at $160^{\circ} \mathrm{C}$. This will, firstly, monitor any possibility of sensitivity changes and, secondly, eliminate or minimize the possible effect of photo-transferred luminescence on the interpretation of the data. Photo-transferred TL is known to play a significant role in these types of materials at elevated temperatures which result in complex continuous wave (CW) decay curves (e.g., McKeever, 2001: Figs 17, 19).

\section{THE EFFECT OF SAMPLE TEMPERATURE ON IRSL $(\lambda>600 \mathrm{~nm})$ SIGNAL AND BACKGROUND}

Both the red IRSL signal and background are temperature-dependent (Fattahi, 2001; 2004). Thermal background is significantly greater than instrumental background for both orange-near-red and far-red (Fig. 1). It is essential to remove from the data, prior to presentation, any background components (including thermal, instrumental and the scattering of IR light and, hence, breakthrough) that are not related to the IR-induced luminescence signals.

To omit background components, the average of the last 10 seconds of the IRSL decay curves (120-130 seconds of the luminescence measurement) were subtracted from all points of IRSL signal between 32-130 seconds (Fig. 2).

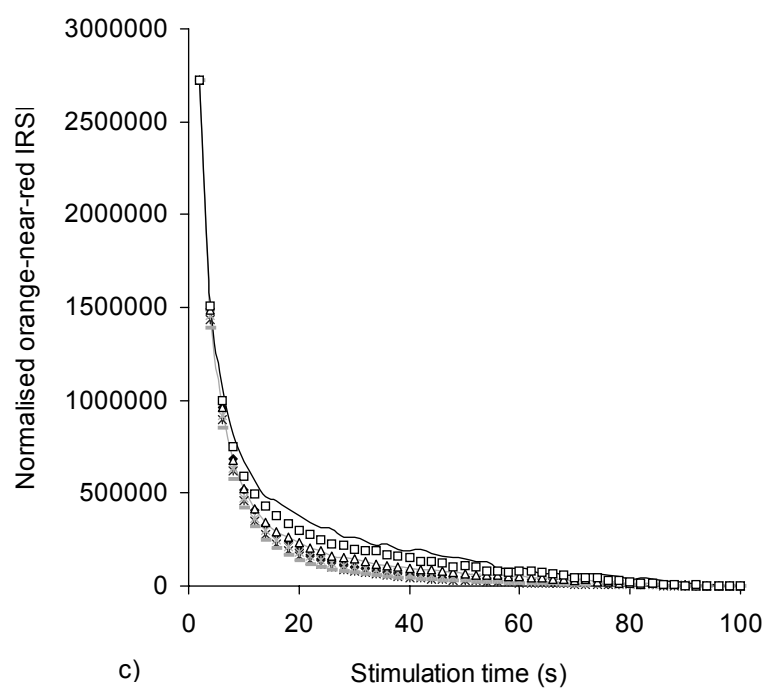

a)
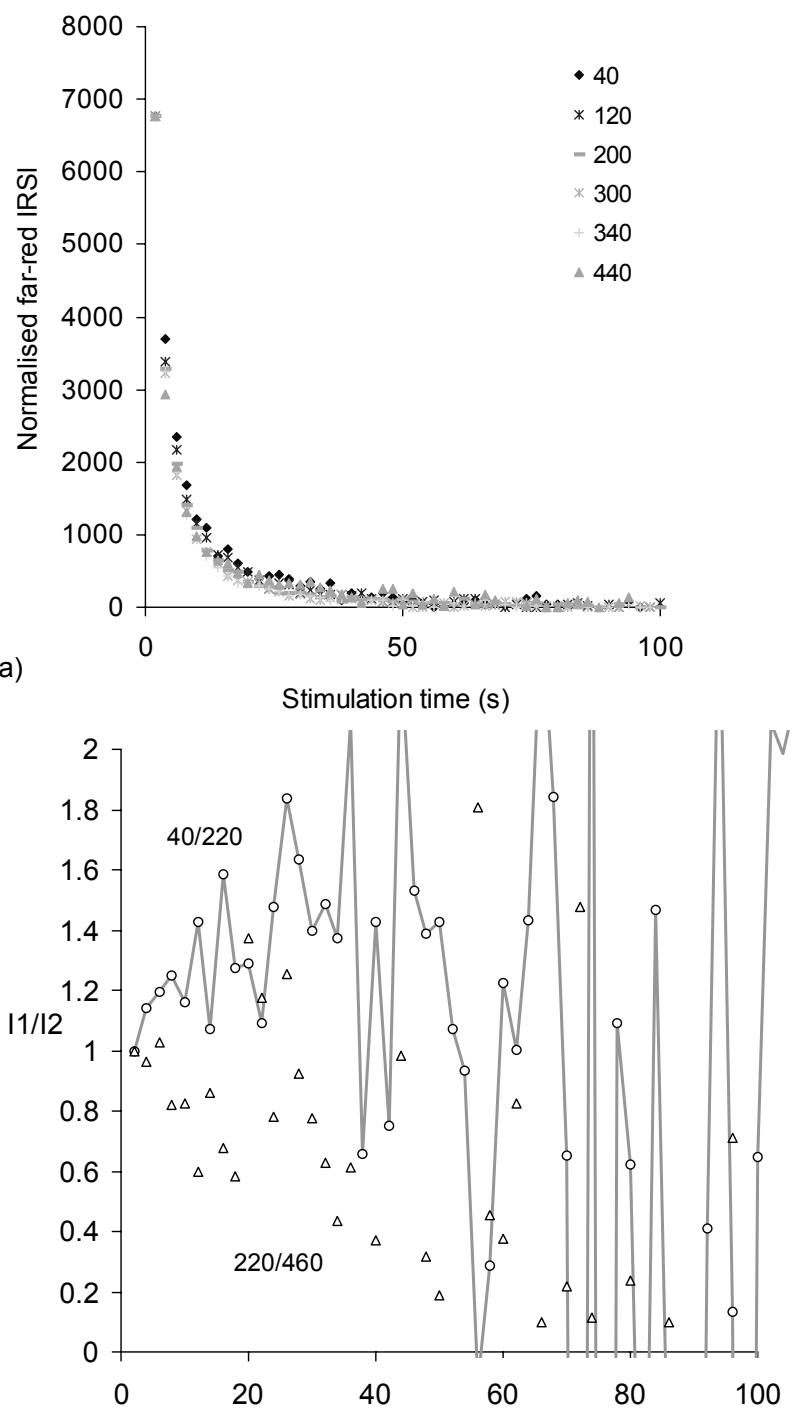

b)

Stimulation time (s)

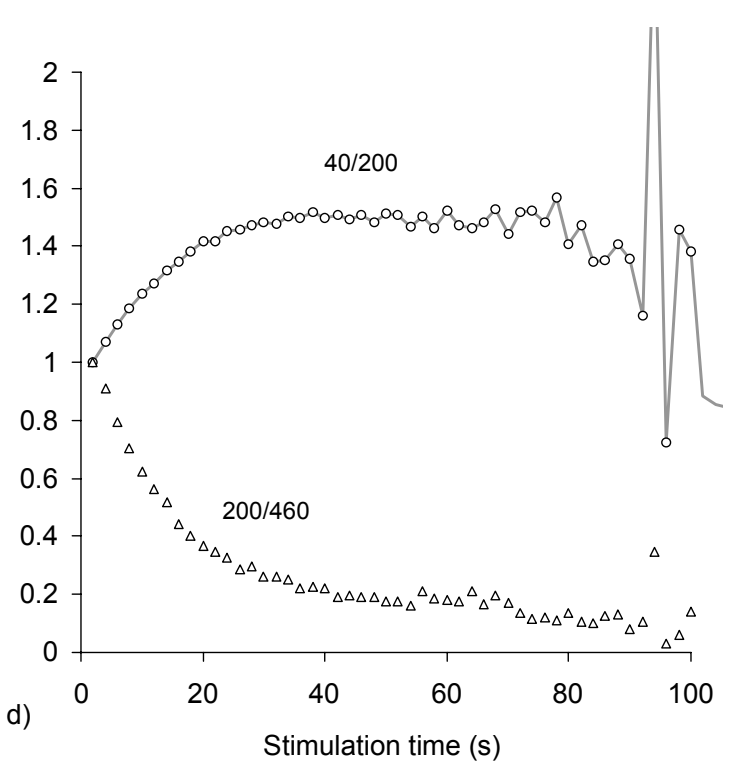

Fig. 2. Normalized IRSL curves for (a) far-red and (c) orange-near-red IRSL signals (see text), from 40 to $460^{\circ} \mathrm{C}$, in $20^{\circ} \mathrm{C}$ steps using IRSL from a prior $2 \mathrm{~s}$ stimulation at $40^{\circ} \mathrm{C}$ for normalization. Inset, shows the difference between the shapes of the decay curves more clearly. The normalized IRSL curve measured at $40^{\circ} \mathrm{C}$ is divided to the IRSL curve measured at $220^{\circ} \mathrm{C}$ for (b) far-red and $200^{\circ} \mathrm{C}$ for (d) near red. And the same as (a) and (c) but only for curves from 40 to $220^{\circ} \mathrm{C}$ that show the rate of the decay is largely affected (as indicated by the ratio of IRSL measured at 40 to and farred, respectively). 
IRSL decay curves and decay rates at elevated temperatures

The shape of the IRSL decay curve depends on several factors, including IR stimulation intensity, detection window and the measurement temperature. Analyzing the IRSL decay curves can increase our knowledge of the mechanisms of trapping and recombination of the charge carriers in feldspar.

Figs $2 \mathbf{a}$ and $\mathbf{c}$ show, a set of normalized IR-stimulated OSL curves for temperatures from 40 to 440 and $460^{\circ} \mathrm{C}$ for far-red and orange-near-red emissions, respectively. Normalization was performed by dividing the signal by the IRSL signal from a prior $2 \mathrm{~s}$ stimulation at $40^{\circ} \mathrm{C}$.

Figs $2 \mathbf{b}$ and $\mathbf{d}$ show that within the first 30 seconds, the rate of the decay has increased up to $50 \%$ for a decay curve measured at $220^{\circ} \mathrm{C}$ (for far-red) and $200^{\circ} \mathrm{C}$ (orange-near-red) decay curves, in comparison to the decay curve measured at $40^{\circ} \mathrm{C}$. This is shown by the ratio of IRSL measured at 40 to $220^{\circ} \mathrm{C}$ and 40 to $200^{\circ} \mathrm{C}$ for farred and orange-near-red, respectively. No systematic change of decay rate can be seen after 30 seconds of IRSL measurements for any substantial temperature.

A trend of decreasing decay rate with increasing temperature is observed within the first 30 seconds of luminescence measurement of this data set from $220-460^{\circ} \mathrm{C}$ and $200-460^{\circ} \mathrm{C}$ for far-red and orange-near-red signals, respectively (Figs $\mathbf{2 b}$ and $\mathbf{d}$ ). This decreasing decay rate by increasing temperature is due to the proportional effect of different components including the effect of background on signal that increases as the sample temperatures increase.

\section{Comparing various portions of the orange-near-red and far-red IRSL decay curves at elevated tempera- tures}

Various parts of the IRSL decay curves presented in Fig. 2 can be plotted as a function of stimulation temperature. Fig. 3 shows the variation in initial (0-2 s), integral (0-100 s), net initial (0-2 s less background over $2 \mathrm{~s}$ (estimated using average light sum of integral from 90$100 \mathrm{~s})$ ) and net integral (0-100 s less background over $100 \mathrm{~s}$ (estimated using average light sum of integral from 90-100 s)) of the IRSL signal for both the far-red (Fig. 3a) and orange-near-red emissions (Fig. 3c).

The measurements show an increase in the far-red IRSL signal in the initial, integral, net initial and net integral between $20-220^{\circ} \mathrm{C}$ (Fig. 3a). The initial, net initial and net integral then decreases between 220$460^{\circ} \mathrm{C}$. When plotted on a logarithmic scale, the initial, net initial, integral and net integral signals increased in parallel up to $220^{\circ} \mathrm{C}$ (Fig. 3b). This suggests that these signals behave in a similar way over these ranges of stimulation temperatures. With data normalized to the far-red IRSL signal obtained at $40^{\circ} \mathrm{C}$, Fig. 3c shows that initial, net initial and net integral match in the temperature range of 240 to $460^{\circ} \mathrm{C}$.

For orange-near-red emissions, a plateau between $100-220^{\circ} \mathrm{C}$ follows an early increase in the IRSL signal in the initial, net initial and net integral between $20-100^{\circ} \mathrm{C}$ (Fig. 3d). It then decreases between $220-460^{\circ} \mathrm{C}$.
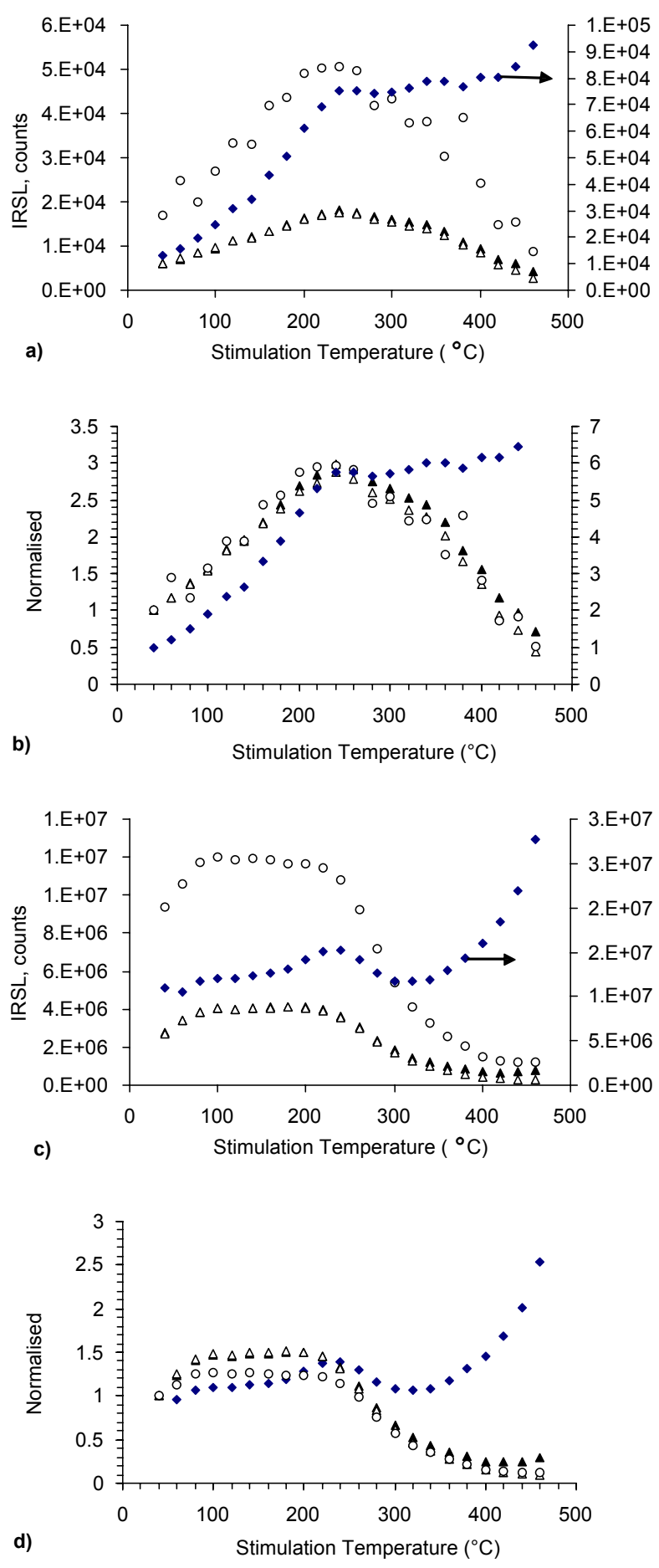

Fig. 3. Variation in initial (0-2 s), integral (0-100s), net initial (0-2 s less background over $2 s$ (estimated using average light sum of integral from 90-100 s)) and net integral (0-100 s less background over $100 \mathrm{~s}$ (estimated using average light sum of integral from 90-100 s)) laboratory irradiated IRSL signals obtained for $100 \mathrm{~s}$ at stimulation temperature plotted versus stimulation temperature. (a) The actual data. (b) Data normalized to unity at $40^{\circ} \mathrm{C}$ for far-red IRSL (c) and (d) the same as (a) and (b), respectively but for orange-near-red IRSL signals. All measurements were performed following preheating for $120 \mathrm{~s}$ at $250^{\circ} \mathrm{C}$.

The net integrated IRSL signals and that of the net initial part of the IRSL are plotted against the stimulation temperature for both orange-near-red and far-red lumi- 


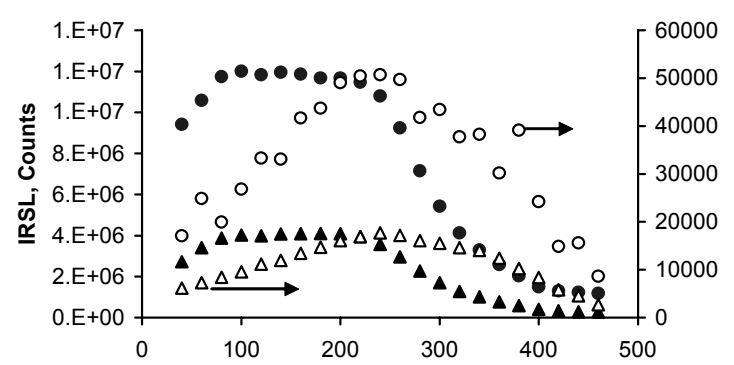

(a)

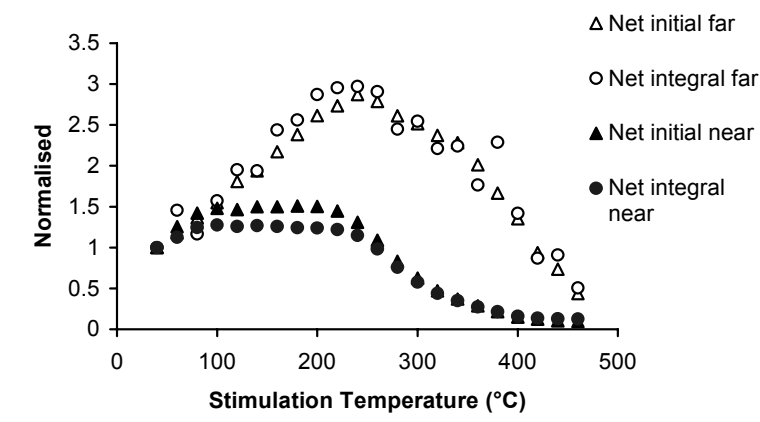

(b)

Fig. 4. Variation in net initial and net integral laboratory irradiated orange-near-red and far-red IRSL signals obtained for $100 \mathrm{~s}$ at stimulation temperature plotted versus stimulation temperature. (a) The actual data. (b) Data normalized to unity at $40^{\circ} \mathrm{C}$.

nescence emissions (Fig. 4a). The number of counts of orange-near-red signals is two to three times greater magnitude than that of far-red signals in the temperature range of $100-300^{\circ} \mathrm{C}$. Both the net integrated and the net initial IRSL signals increase (Fig. 4) with temperatures in the range from $20-100^{\circ} \mathrm{C}$ and $20-220^{\circ} \mathrm{C}$ for orange-nearred and far-red emissions, respectively. This increase in the net initial or net integral orange-near-red IRSL at low temperatures agrees with the previous studies on optically stimulated blue emissions of feldspars (e.g., Duller and Wintle, 1991; Rieser et al., 1997).

With data normalized to the IRSL signals obtained at $40^{\circ} \mathrm{C}$, we can compare the behaviour of net initial and net integrated signals in the orange-near-red and far-red emissions (Fig. 4b). The net integrated and the net initial part of the far-red IRSL and orange-near-red signals match with each other between 40 to $80^{\circ} \mathrm{C}$. This suggests that the net initial and the net integral, follow the same mechanisms in the sample temperature range of 40 to $80^{\circ} \mathrm{C}$ for both near-orange-red and far-red IRSL signals.

The orange-near-red values are almost constant over the temperature range 100 to $220^{\circ} \mathrm{C}$, while the far-red values systematically increase. This difference is related to the effects of thermal phenomena. The flat pattern of orange-near-red IRSL can be due to the equal competition between thermal assistant and the combination of thermal depletion and thermal quenching. For far-red, the effect of thermal enhancement is much greater than the effect of the combination of thermal depletion and thermal quenching. This increase of the net initial and net integral IRSL in far-red emissions (Fig. 4) may be due to several factors including: (a) Contrasting mechanisms of IRSL production for far-red in comparison with orange- near red IRSL. (b) The orange-near red peak is comprised of a combination of the left tail of far-red and the right tail of the yellow emission (with a peak $\sim 560 \mathrm{~nm}$ ), coupled with different mechanisms of production for the two emissions. (c) The combined effect of thermal enhancement, quenching and depletion is different for far-red and orange-near-red (e.g., far-red IRSL thermal quenching is negligible in comparison to thermal enhancement, while for orange-near-red this is not the case). (d) For far-red more luminescence centres are available at higher temperatures and temperature dependence of photo evicted charges increase by increasing temperature. (e) For farred IRSL there is an additional source of charge at higher temperatures that originate from a deeper trap or traps including thermal transfer of charges from thermally sensitive, but not optically sensitive deeper traps to the optically sensitive shallower traps (in comparison to orange-near-red emissions), so the effect of thermal depletion commences at temperatures higher than say $100^{\circ} \mathrm{C}$.

The normalized values of both net initial and net integral of far-red IRSL signals are almost similar in Fig. $\mathbf{4 b}$. The same scenario is observable for orange-near-red IRSL signals. This similarity in the range of $100-240^{\circ} \mathrm{C}$ suggests that thermal quenching is minimal for both farred and orange-near-red IRSL signals in this range of temperature.

The net initial and net integral of orange-near-red and far-red IRSL signals decrease at $220^{\circ} \mathrm{C}$ and $240^{\circ} \mathrm{C}$, respectively (Fig. 4b). The range of $220-240^{\circ} \mathrm{C}$ is the critical temperature at which depletion, produced by the $30 \mathrm{~s}$ heating, at the measurement temperature prior to stimulation, prevails over thermal enhancement for both orangenear-red and far-red signals. This suggests that electrons responsible for producing both far and near red IRSL signals may originate from the same electron traps. However, the decay pattern for the far-red IRSL signals is different from the one of orange-near-red signals for the temperature range of 220 to $460^{\circ} \mathrm{C}$ (Fig. 4b). This suggests that the far-red IRSL and orange-near-red signals follow different mechanisms in the sample temperature range of 260 to $460^{\circ} \mathrm{C}$.

However, far-red IRSL signals (for both net initial and net integral) have two significant differences with the orange-near-red IRSL signals. Firstly, far-red IRSL signals increase in the temperature range between 100 $220^{\circ} \mathrm{C}$, while orange-near-red IRSL signals possess a constant value. Secondly, although all signals are decreasing in temperature range between $240-460^{\circ} \mathrm{C}$, the form of the decay of far-red (net initial and net integral) signals is different from the form of decay of the orangenear-red signals. This suggests that two different physical mechanisms or pathways may govern the movement of electrons to produce luminescence in this feldspar sample (one for far-red and another for orange- near-red signals).

\section{Calculation of $E$}

\section{Thermal assistance energy}

The data of Fig. 3 can be used directly to calculate the energy of thermal assistance $(E)$, using an Arrhenius plot 
of the variation in the orange-near-red and the far-red IRSL. Arrhenius plot is based on:

$$
I(t, T) \approx \exp \left(-\frac{E}{k T}\right) I(t)
$$

Where $\mathrm{k}$ is the Boltzmann constant and $\mathrm{E}$ is the measured activation energy. Figs 5a and $\mathbf{b}$ show Arrhenius plots of both the initial and the integral signals against $1 / \mathrm{kT}$, of the far-red IRSL and orange-near-red IRSL intensity, respectively. Average $E$ of $0.076 \pm 0.034 \mathrm{eV}$ and $0.015 \pm 0.001 \mathrm{eV}$ are calculated for far-red and orangenear-red, respectively (Table 1).

\section{Thermal depletion energy of orange-near-red lumines- cence}

The experimental data between $220-280^{\circ} \mathrm{C}$, can be used to calculate the energy of thermal depletion, $E_{d}$ using isothermal decay observed in the first 30 seconds of luminescence measurement (before illumination). An equation of the form:

$I(t)=I_{0} \exp \left(-\frac{t}{\tau}\right)$

provides the simplest representation of the data (assuming first order kinetics), where $\mathrm{I}(\mathrm{t})$ is the intensity of the luminescence at time $\mathrm{t}, \mathrm{I}_{0}$ is the intensity at $\mathrm{t}=0$ and $\tau$, the duration over which an electron can be expected to remain trapped, is the lifetime, and is given by

$$
\tau=s^{-1} \exp \left(\frac{E}{k T}\right)
$$

where $s$, is the frequency factor, $E$, the trap depth: $k$, the Boltzmann constant, and $T$, the absolute temperature, are constant.

Values of $\tau$ were estimated from the normalized isothermal decay data for the temperatures used. The natural logarithms of the calculated life times are plotted as a function of storage temperature for the exponential decaying component that yield a straight line with a slope of $E$ and the intercepts of $1 \mathrm{n}(\mathrm{s})$, (Fig. 6). The values of $E_{m}$ and $s$ are $E_{m}=1.15 \pm 0.05 \mathrm{eV}$ and $s=(3.64 \pm 0.02) \cdot 10^{8} \mathrm{~s}^{-1}$.

Thus, the $E$ value can be interpreted as the energy of thermal ionization of some centres (e.g., the lowtemperature component of the dosimetric levels associated with the first TL doublet in the $240^{\circ} \mathrm{C}$ region, see Duller, 1997). The $E$ value obtained as ca $1.1 \mathrm{eV}$ can correspond to the $\mathrm{TL}$ peak at $\mathrm{T}<200^{\circ} \mathrm{C}$. The possible reason for the contradiction is the possibility of thermally unstable centres and the kinetics of the process that does not match the first order. It should be mentioned that if in the given temperature area $\left(220-280^{\circ} \mathrm{C}\right)$ there is a thermal quenching, then:

$E($ experimental $)=E($ true $)-E($ quenching $)$

where $E$ (quenching) is the activation energy of thermal quenching.

\section{DISCUSSION}

Figs 3 and 4 show that greater IRSL signals are obtained at an elevated temperature $\left(220^{\circ} \mathrm{C}\right.$ for far-red and $100^{\circ} \mathrm{C}$ for orange-near-red) than are obtained at a lower
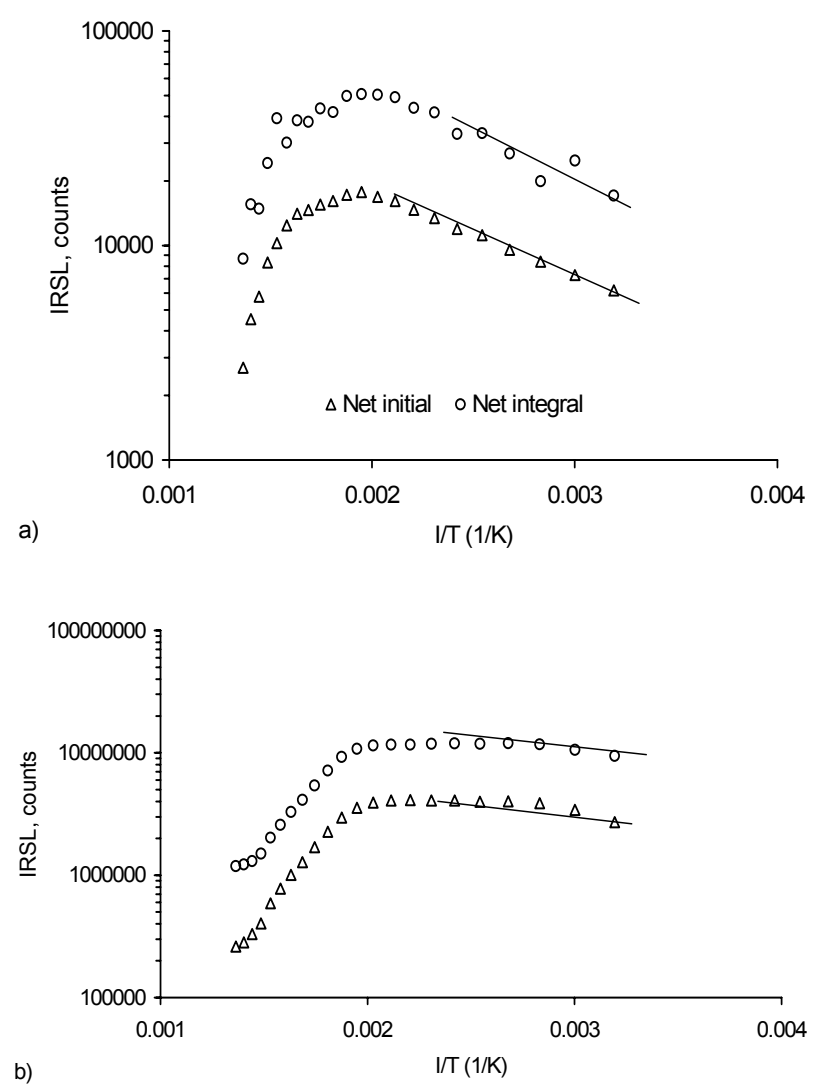

Fig. 5. Arrhenius plot for data from Fig. 4. The net initial and net integral vs. 1/T ( $T$ =stimulation temperature) (a) for far-red, (b) for orangenear-red. (0.00136) to $(0.00319)$ corresponds to $460^{\circ} \mathrm{C}-40^{\circ} \mathrm{C}$, respectively.

temperature $\left(40^{\circ} \mathrm{C}\right)$. The Hütt model may interpret the cause of increase in the far-red IRSL intensity up to 30 seconds, as sample temperature increases from $20-220^{\circ} \mathrm{C}$.

However, in the Hütt model, thermal assistance usually affects the rate of optical ejection of charge, and will therefore affect the net initial signal. The net integral in this case should not be affected by changes in the rate of IRSL decay. Fig. 4b, however, shows that both the net initial and the net integral IRSL signals increase with sample temperature and sets of normalized far-red IRSL decay curves observed in Fig. 2b do not show any trend of increasing decay rate with increasing temperature after 30 seconds, as expected from a Hütt model. So the Hütt model could not explain the cause of increase in far-red IRSL intensity after 30 seconds, as the sample temperature increases from $20-220^{\circ} \mathrm{C}$. Red IRSL could be interpreted as resulting from the elevation of the charges to an excited state by acquiring thermal energy from the lattice, and subsequent elevation to the conduction band by the IR excitation (IR transition). UV-blue luminescence is interpreted as optically prompting the charge to an excited state, and subsequently elevated to the conduction band by thermal energy from the lattice. In red IRSL, thermal assistance may occur before the IR excitation; therefore thermal assistance does not affect the decay curve, and affects the net initial and net integral signal similarly, as has been observed in Fig. 4. The thermal activation energy measured for the red IRSL, therefore, 
Table 1. Thermal Activation Energy of far-red and near-red IRSL.

\begin{tabular}{llllll}
\hline Disk & Temperature $\left({ }^{\circ} \mathrm{C}\right)$ & Emission & Net first & Net integral & Average \\
\hline 47 & $20-260$ & far-red & 0.077 & 0.085 & $0.076 \pm 0.034$ \\
42 & $20-260$ & far-red & 0.066 & 0.067 & \\
45 & $20-260$ & far-red & 0.074 & 0.084 & 0.072 \\
1 & $40-240$ & far-red & 0.084 & & $0.015 \pm 0.001$ \\
47 & $20-240$ & & & 0.014 & 0.016 \\
42 & $20-180$ & near-red & 0.014 & 0.016 & \\
\hline
\end{tabular}

will be the thermal eviction of charge from the trap to the excited state. However, there is no evidence to confirm or refute this mechanism and further study is required.

Another possible model that may interpret these observations is as follows: there is continuum of traps which give rise to IRSL. During IRSL measurement, the depth of the traps accessed by the IR stimulation is temperature dependent, i.e. at higher temperatures more traps (deeper traps) are accessible. To examine this hypothesis, a model should be examined and tested in which the IRSL signal is the sum of the exponential decays from all the traps that are active at the measurement temperature, plus the background.

In the temperature range of $40-220^{\circ} \mathrm{C}$ the $0.076 \mathrm{eV}$ assists the far-red IRSL, whereas the orange-near-red IRSL is assisted by lower energetic phonons $(0.015 \mathrm{eV})$ (Fig. 5; Table 1). This suggests the possible existence of two separate physical mechanisms for orange-near-red IRSL and far-red IRSL. Rieser et al. (1997) calculated the thermal activation energy $(0.05-0.15 \mathrm{eV})$ between -130 and $200^{\circ} \mathrm{C}$ for both blue and yellow emissions. Therefore, the lower value of $0.015 \pm 0.001 \mathrm{eV}$ for orange-near-red in the temperature area above $100^{\circ} \mathrm{C}$ might not be the true $E$ value. For example, if the sample suffered from thermal quenching (e.g., see Duller, 1997) then equation (4.4) may be used. If this is true then there may be no particular reasons for the assumption that in orange-red and farred luminescence bands mechanisms of IRSL occurrence are basically different.

Could far-red IRSL be interpreted as a band tail states model? Poolton et al. (2002b) suggested that the presence of band-tail states below the conduction band could explain the thermal dependence of IRSL. They argued that general temperature dependence form (Poolton et al. 2002b: Equation 9) suggests that the phonons likely to play a part in the model are those with energies at 0.04$0.05 \mathrm{eV}$ that lies in a region of strong lattice vibration. These energies are, however, not far from thermal activation energy for far-red IRSL (Table 1) that lie between 0.07 and $0.08 \mathrm{eV}$. Therefore, experimental observations outlined above can be interpreted by the random-walk band-tail model of Poolton et al. (2002b).

Fattahi and Stokes $(2003 \mathrm{~b}, \mathrm{c})$ have shown that far-red is more stable than orange-near-red IRSL signal. Therefore, the far-red IRSL process might be interpreted as non-tunnelling, while orange-near-red can be interpreted as a tunnelling process.
It should be mentioned that after each main luminescence measurement at the elevated temperature, sensitivity changes were monitored by the response of a test dose (Fig. 7a). Sensitivity changes were corrected by dividing the net initial signal of the regenerated by the net initial of the test dose signal. The sensitivity did not change the pattern of signal up to $240^{\circ} \mathrm{C}$ for both emissions (Figs $7 \mathbf{b}$, c). Therefore, we can assume that what this paper has discussed is related to pure IRSL signals at the elevated temperature and not to unwanted phenomena, at least for sample temperatures up to $240^{\circ} \mathrm{C}$.

\section{CONCLUSION AND FUTURE DIRECTION}

There are similarities between orange-near-red and far-red IRSL signals from identical feldspar samples. For net normalized IRSL decay curves, the decay rate does not change significantly after 30 seconds in the temperature range $40-340^{\circ} \mathrm{C}$ for both emissions (Figs $\mathbf{2 b}$ and $\mathbf{d}$ ). There is signal enhancement for both emissions in the temperature range $20-100^{\circ} \mathrm{C}$. There is signal reduction in the temperature range from 260 to $460^{\circ} \mathrm{C}$ for both emissions (Fig. 4). This study suggests that electrons responsible for producing both far and orange-near-red IRSL signals originate from the same electron traps.

There are also differences between orange-near-red and far-red IRSL signals. While far-red IRSL signals increase, orange-near-red IRSL signals do not change in the temperature range $100-220^{\circ} \mathrm{C}$ (Fig. 4). Although all

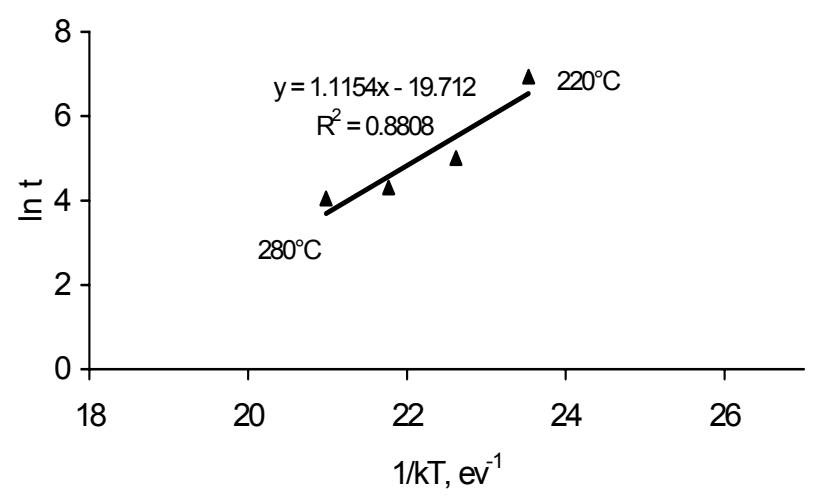

Fig. 6. The natural logarithms of the calculated life times are plotted as a function of $1 / \mathrm{k} T$ ( $T=$ stimulation temperature) for orange-near-red. 


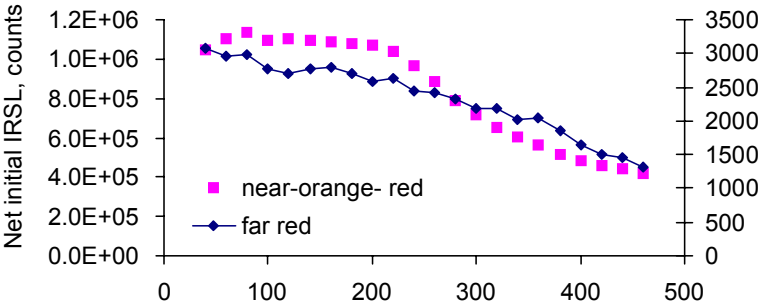

a)

Sample Temperature of previous measurement $\left({ }^{\circ} \mathrm{C}\right)$
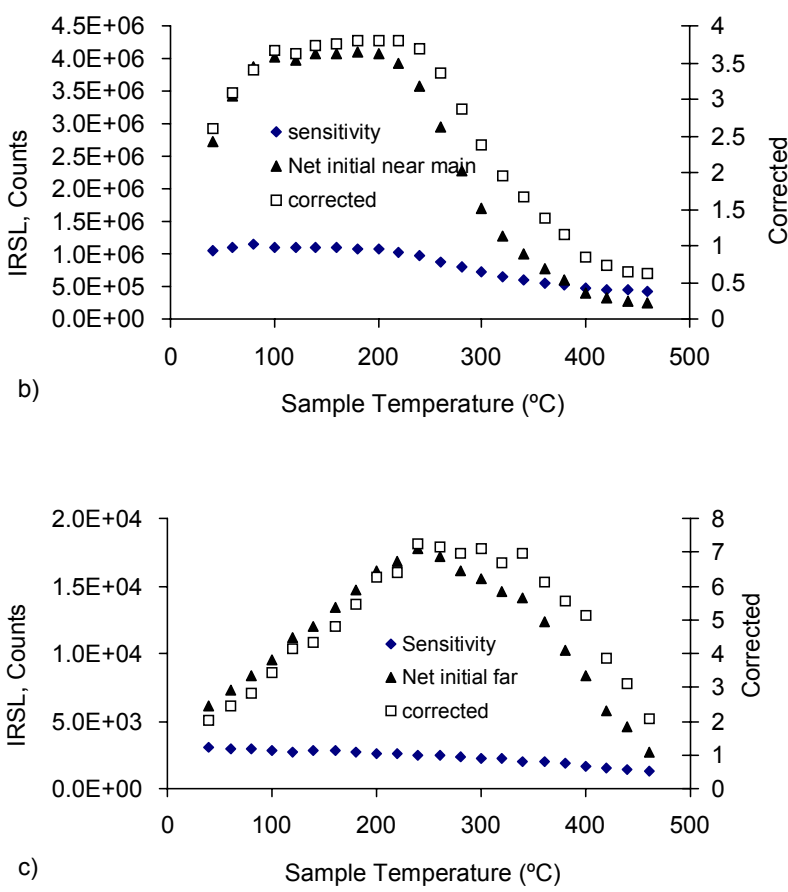

Fig. 7. a) Net initial of IRSL responses to test dose (60 Gy), measured at $160^{\circ} \mathrm{C}$ for 300 seconds. Following regenerated luminescence measurements at elevated temperature. b) Net initial of regenerated and corrected signals versus sample temperature for orange-red IRSL. c) The same as b) but for far-red signals.

signals are decreasing in the temperature range between $240-460^{\circ} \mathrm{C}$, the form of the decay of far-red signals is different from the form of decay of the orange-near-red signals. In the temperature range of $40-220^{\circ} \mathrm{C}$ the $0.076 \mathrm{eV}$ assists far-red IRSL, whereas orange-near-red IRSL is assisted by much less energetic phonons $(0.015 \mathrm{eV})$ (Fig. 5). As far-red IRSL signals of this sample seem more stable than the orange-near-red IRSL (Fattahi, 2001), the difference in activation energy for both emissions suggest that the tunnelling and nontunnelling may co-exist in this sample.

The similarity and differences between orange-nearred and far-red IRSL signals suggest that different physical mechanisms or pathways govern the movement of electrons to produce luminescence in this feldspar sample. These mechanisms are examined via interpretation of data in both orange-near-red and far-red emission bands. Among them the experimental observations suggest that the free-to-bound recombination and direct electron-hole tunnelling recombination might be more relevant or the dominant processes to interpret our findings in the temperature range $20-220^{\circ} \mathrm{C}$ for far-red and orange-near IRSL, respectively.

However, many examples of luminescence do not involve charge transport through the conduction band but are rather the results of charge movements and relaxations within extended defect complexes within the material. This is particularly likely for a material such as feldspar which has variations in composition and not "perfect" long range crystal structure. Band models for luminescence seem too simplistic.

Further research is essential to provide sufficient information to enable us to interpret the experimental results more precisely. Such research requires first, employing well-behaved samples with known mineralogy; and second, extending the experiments for completely separated emissions bands (UV, Blue, Yellow, Orange-Red and Far-Red). Thirdly, it requires extending the experiments for various kinds of feldspars.

\section{ACKNOWLEDGEMENTS}

The Research Department of Tehran University provided my travel expenses for this project. The School of Geography of Oxford University Centre of Environment has provided all the experimental facilities and requirements. The author would like to thank Alison Colls who provided the feldspar sample 15/1; Genine Natale who improved the general language of the paper and Gita Nejad for general support. I am grateful to both reviewers and editorial boards for their positive suggestions, comments and advice, which resulted in a significantly improved final manuscript.

\section{REFERENCES}

Aitken MJ, 1998. An Introduction to Optical Dating: The dating of Quaternary sediments by the use of photon-stimulated luminescence. Oxford, Oxford University Press: $267 \mathrm{pp}$.

Bailiff IK and Barnett SM, 1994. Characteristics of infrared stimulated luminescence from feldspar at low temperature. Radiation Measurements 23: 541-545, DOI 10.1016/1350-4487(94)90096-5.

Bøtter-Jensen L, Duller GAT and Murray AS, 2000. Advances in luminescence instrument systems. Radiation Measurements 32(56): 523-528, DOI 10.1016/S1350-4487(00)00039-1.

Clarke ML, Wintle AG and Lancaster N, 1996. Infra-red stimulated luminescence dating of sands from the Cronese Basins, Mojave Desert. Geomorphology 17(1-3): 199-205, DOI 10.1016/0169. 555X(95)00103-C.

Colls AEL, 1999. Optical dating of fluvial sediment from the Loire Valley, France. MSc Thesis. Oxford, University of Oxford.

Colls AE, Stokes S, Blum MD, Straffin E, 2001. Age limits on the late quaternary evolution of the upper Loire River. Quaternary Science Reviews 20: 743-750, DOI 10.1016/S0277-3791(00)00048-2.

Deer WA, Howie RA and Zussman J, 1992. An Introduction to the Rock-Forming Minerals. Longman, Harlow: 696 pp.

Duller GAT, 1994. Luminescence dating using feldspars: a test case from southern North Island in New Zealand. Quaternary Science Reviews 13: 423-427, DOI 10.1016/0277-3791(94)90053-1.

Duller GAT, 1997. Behavioural studies of stimulated luminescence from feldspars. Radiation Measurements 27: 663-694, DOI 10.1016/S1350-4487(97)00216-3.

Duller GAT and Wintle AG, 1991. On infra-red stimulated luminescence at elevated temperatures. Nuclear Tracks and Radiation Measurements 18(D: 4): 379-384, DOI 10.1016/1359. 0189(91)90003-Z. 
Fattahi M, 2001. Studies on red thermoluminescence and infrared stimulated red luminescence. Unpublished D. Phil. Thesis. Oxford, Oxford University.

Fattahi M, 2004. The dependence of orange-red IRSL decay curves of potassium feldspar on sample temperature. Radiation Measurements 38(3): 287-298, DOI 10.1016/j.radmeas.2003.09.006.

Fattahi M and Stokes S, 2003a. Photomultiplier and filter combinations for the detection of relatively long wavelength $(\lambda>600 \mathrm{~nm})$ luminescence emissions from feldspar. Ancient TL 21(1): 25-35.

Fattahi M and Stokes S, 2003b. Infrared stimulated red luminescence from potassium feldspar for dating applications: A study of some properties relevant for dating. Radiation Measurements 37(6): 647-660, DOI 10.1016/S1350-4487(03)00246-4.

Fattahi M and Stokes S, 2003c. Absorbed dose evaluation in feldspar using a single-aliquot regenerative-dose (SAR) infrared-stimulated red luminescence protocol. Radiation Measurements 38(1): 127134, DOI 10.1016/S1350-4487(03)00248-8.

Fattahi M, Walker R, Hollingsworth J, Bahroudi A, Talebian M, Armitage S, and Stokes S, 2006. Holocene slip-rate on the Sabzevar thrust fault, NE Iran, determined using Optically-stimulated Luminescence (OSL). Earth and Planetary Science Letters 245: 673684, DOI 10.1016/j.eps1.2006.03.027.

Hütt G and Jaek I, 1993. Photostimulated luminescence of some materials and its dosimetry applications. Nuclear Tracks and Radiation Measurements. Part D 21(5-6): 95-98, DOI 10.1016/13590189(93)90051-A.

Hütt G, Jaek I and Tchonka J, 1988. Optical dating: potassium feldspars optical response stimulation spectra. Quaternary Science Reviews 7: 381-385, DOI 10.1016/0277-3791(88)90033-9.

Krbetschek MR, Götze J, Dietrich A and Trautmann T, 1997. Spectral information from minerals relevant for luminescence dating. $R a$ diation Measurements 27(5-6): 695-748, DOI 10.1016/S13504487(97)00223-0.

Lamothe M and Auclair M, 1999. A solution to anomalous fading and age shortfalls in optical dating of feldspar minerals. Earth and Planetary Science Letters 171(3): 319-323, DOI 10.1016/S0012821X(99)00180-6.

Lian OB and Shane PA, 2000. Optical dating of paleosols bracketing the widespread Rotoehu tephra, North Island, New Zealand. Quaternary Science Reviews 19: 1649-1662, DOI 10.1016/S02773791(00)00003-2.

Manning PG, 1970. Racah parameters and their relationship to lengths and co-valencies of $\mathrm{Mn}^{2+}$ - and $\mathrm{Fe}^{3+}$-oxygen bonds in silicates. Can Miner 10: 677-688.

McKeever SWS, 2001. Optically stimulated luminescence dosimetry. Nuclear Instruments and Methods B 184: 29-54, DOI 10.1016/S0168-583X(01)00588-2.
McKeever SWS, Bøtter-Jensen L, Agersnap Larsen N and Duller GAT, 1997. Temperature dependence of OSL decay curves: experimental and theoretical aspects. Radiation Measurements 27: 161-170, DOI 10.1016/S1350-4487(96)00106-0.

Molodkov A, Jaek I and VasilchenkoV, 2007. Anomalous fading of IRstimulated luminescence from feldspar minerals: some results of the study. Geochronometria 26: 11-17, DOI 10.2478/v10003-0070007-0.

Poolton NRJ, Bøtter -Jensen L and Johnsen O, 1995. Thermo-optical properties of optically stimulated luminescence in feldspars. $R a$ diation Measurements 24: 531-534, DOI 10.1016/13504487(94)00114-G.

Poolton NRJ, Wallinga J, Murray A, Bulur E and Botter-Jensen L, 2002a. Electrons in feldspar I: on the wavefunction of electrons trapped at simple lattice defects. Physics Chemistry Minerals 29: 210-216, DOI 10.1007/s00269-001-0217-3.

Poolton NRJ, Ozanyan KB, Wallinga J, Murray A and Botter-Jensen L, 2002b. Electrons in feldspar II: a consideration of the influence of conduction band-tail states on luminescence processes. Physics Chemistry Minerals 29: 217-225, DOI 10.1007/s00269-001-02182.

Richardson CA, McDonald EV and Busacca AJ, 1999. A luminescence chronology for loess deposition in Washington State and Oregon, USA. Zeitschrift fur Geomorphologie 116: 77-95.

Rieser U, Hütt G, Krbetschek MR and Stolz W, 1997. Feldspar IRSL emission spectra at high and low temperatures. Radiation Measurements 27: 273-278, DOI 10.1016/S1350-4487(96)00108-4.

Telfer DJ and Walker G, 1978. Ligand field bands of $\mathrm{Mn}^{2+}-$ and $\mathrm{Fe}^{3+}$ luminescence centers and their site occupancy in plagioclase feldspars. Modern Geology 6: 199-210.

Trautmann T, Krbetschek MR, Dietrich A and Stolz W, 2000. The basic principle of radioluminescence dating and a localized transition model. Radiation Measurements 32(5-6): 487-492, DOI 10.1016/S1350-4487(00)00119-0.

Visocekas R, 2000. Monitoring anomalous fading of TL of feldspars by using far-red emission as a gauge. Radiation Measurements 32: 499-504, DOI 10.1016/S1350-4487(00)00054-8.

Visocekas R, Tale V, Zink A, Spooner NA and Tale I, 1996. Trap spectroscopy and TSL in feldspars. Radiation Protection Dosimetry 66(1-4): 391-394.

Wallinga J, Murray AS, Duller G, 2000. Underestimation of equivalent dose in single-aliquot optical dating of feldspars caused by preheating. Radiation Measurements 32(5-6): 691-695, DOI 10.1016/S1350-4487(00)00127-X.

Zink AJC and Visocekas R, 1997. Datability of sanidine feldspars using the near-infrared TL emission. Radiation Measurements 27(2): 251-261, DOI 10.1016/S1350-4487(96)00141-2. 